Fizika Nizkikh Temperatur, 2000, v. 26, Nos. 9/10, p. 937-946

\title{
EXAFS studies of the trapping site structure for molecules isolated in cryogenic matrices
}

\author{
P. Roubin, S. Varin \\ P2IM, Université de Provence, Centre Saint-Jérôme, 13397 Marseille cedex 20, France \\ C. Crépin
}

LPPM, Bât 213, Centre Universitaire Paris-Sud, 91898 Orsay cedex, France

B. Gauthier-Roy

LPMA, Université P. et M. Curie, 4 Place Jussieu, 75252 Paris cedex 05, France

\author{
A.-M. Flank, P. Lagarde, and F. Ténégal \\ Lure, Bât 209D, Centre Universitaire Paris-Sud, 91898 Orsay cedex, France
}

Received May 11, 2000

\begin{abstract}
We present here results concerning the first attempt of determining the trapping site structure of molecules isolated in inert matrices at low temperature by the EXAFS (Extended X-ray Absorption Fine Structure) method. The experiments have been performed at the $K$ edge of argon, silicon, sulfur, and chlorine for pure solid argon, and for $\mathrm{SiH}_{4}$, OCS and $\mathrm{HCl}$ isolated in different cryogenic matrices. The EXAFS technique is sensitive to the local environment around the absorbing atom and the spectral features induced by the matrix material ( $\mathrm{Ar}, \mathrm{Xe}, \mathrm{N}_{2}$ and $\mathrm{CH}_{4}$ ) are clearly evidenced here. The data allow a characterization of the double substitutional site for OCS in argon and xenon while no structure can be determined for the accommodation of $\mathrm{SiH}_{4}$ in argon. A discussion of the best choice for the guest/host system to obtain a good EXAFS signal is included.
\end{abstract}

PACS: $33.20 . \mathrm{Rm}, 61.10 . \mathrm{Ht}$

\section{Introduction}

Isolating molecules in cryogenic and inert matrices usually leads to simplified spectra because of the absence of a rotational structure but these spectra often show fingerprints due to the weak interaction existing with the solvent. The question then arising is about the nature of the trapping cage. Generally, using spectroscopic methods is not straightforward for obtaining a direct comparison between a calculated structure and the experimental observations. Other techniques as x-rays or neutron diffraction are more suitable to get structural information directly, but these methods are sensible to the long range order in the matrix rather than to the local environment around the molecular impurity [1-3].
X-ray absorption is another way to obtain structural information about the environment: as a matter of fact, the $\mathrm{x}$-ray absorption spectra are deeply modified when going from the gas phase to the condensed phase and are sensitive to the nearest neighbors surrounding the absorbing atom. The low-frequency part of the spectrum is called XANES (X-ray Absorption Near-Edge Structure) and contains information about the electronic structure, while the high-frequency part of the spectrum is called EXAFS (Extended X-ray Absorption Fine Structure) and gives directly the distances between the absorbing atom and its neighbors. We will present in part 2 an introduction to the basics 


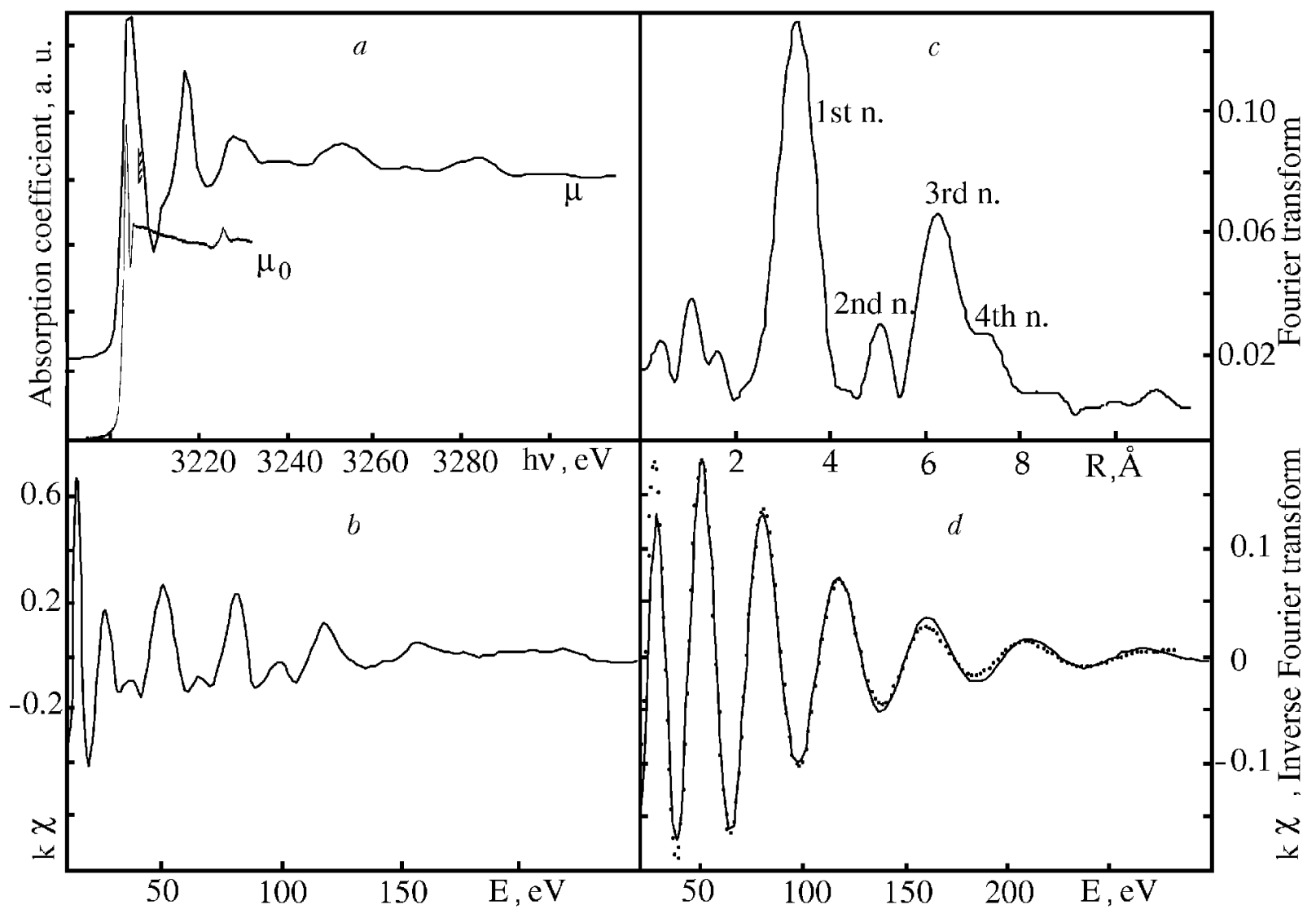

Fig. 1. X-ray absorption at the $K$ edge of solid argon. (a) solid argon $(\mu)$; gas phase [7] $\left(\mu_{0}\right)$, the hatched lines indicate the ionization limit; $(b)$ EXAFS oscillations $(k \chi)$ as a function of the photoelectron kinetic energy; (c) Fourier transform of the EXAFS oscillations; $(d)$ inverse Fourier transform of the first neighbor peak: the dots correspond to the experimental data and the continuous line corresponds to a fit with the electronic parameters given by the FEFF code.

concerning the way to obtain the structural information by this latter method.

Previous EXAFS experiments concerning species isolated in matrices are focussed on the study of the species structure itself. In some of these studies [4,5], a distance related to the matrix cage has also been measured. The only work fully devoted to cryogenic inert matrix structure concerns different rare gas and nitrogen matrices [6]. The authors performed EXAFS measurements on solid xenon, krypton, argon and on mixed rare gas matrices, at the $K$ edge of $\mathrm{Ar}(3203 \mathrm{eV})$ and $\mathrm{Kr}(14330 \mathrm{eV})$ and at the $L_{3}$ edge of $\mathrm{Xe}(4782 \mathrm{eV})$. They were able to compare the miscibility of xenon and krypton in argon, neon and nitrogen matrices and they measured a $\mathrm{Kr}-\mathrm{Ar}$ distance of $3.82 \AA$ for diluted $\mathrm{Kr}$ in $\mathrm{Ar}$, intermediate between the pure argon and the pure krypton ones, and close to the free $\mathrm{ArKr}$ molecule value.

The experiments presented in this paper are the first attempt of characterizing the environment of a molecule trapped in a cryogenic medium by the EXAFS technique. We will review here the results obtained for molecules ( $\mathrm{OCS}, \mathrm{SiH}_{4}$ and $\mathrm{HCl}$ ) in rare gas, nitrogen and methane matrices, excited at the $K$ edge of an atom belonging to the molecule $(\mathrm{S}$, $\mathrm{Si}$ and $\mathrm{Cl}$ ). Up to now, we have obtained both qualitative and quantitative results and we will point out the main criteria to be considered in order to get the best advantage of this kind of experiments.

\section{Introduction to the basics of $x$-ray absorption in the condensed phase}

Figure $1, a$ shows the $\mathrm{x}$-ray absorption spectrum of argon in the gas phase [7] $\left(\mu_{0}\right)$ and in the solid phase $(\mu)$. This last spectrum has been obtained by us and is in good agreement with previously published results [8]. It is recorded as a function of the photon energy $(h v)$ for the excitation of an electron belonging to the $K$ shell $(n=1)$ of the argon atom at energy $E_{0} \approx 3202 \mathrm{eV}$.

The first intense line of the gas phase spectrum is due to the absorption towards the $4 p$ Rydberg state ( $1 s \rightarrow 4 p$ dipole-allowed transition), the weaker 


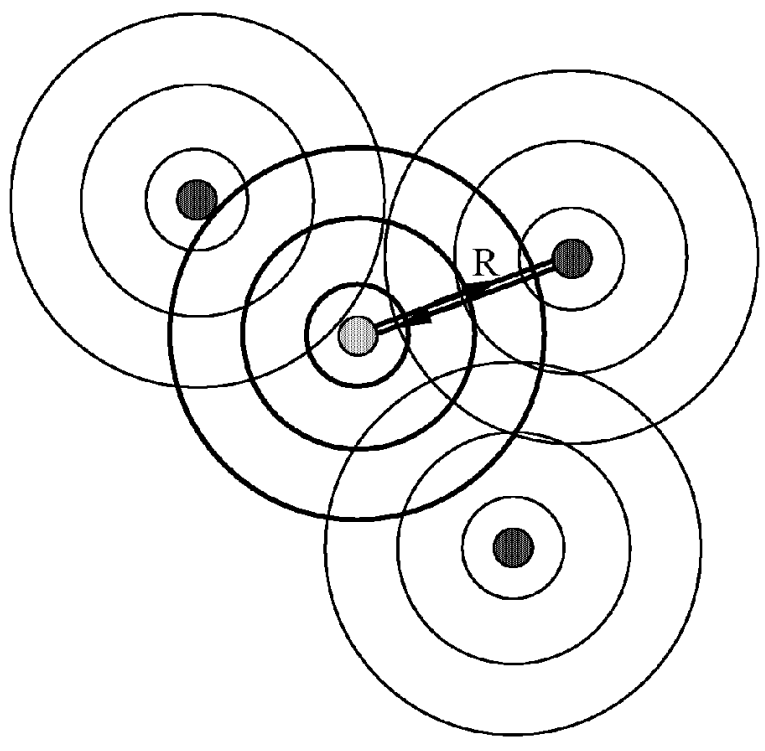

Fig. 2. Schematic of the EXAFS interferences: the bold circles represent the electronic direct outgoing wave (wavenumber $k$ ) coming from the absorbing atom which interferes with the different scattered waves coming from its neighbors. The corresponding optical phase shift is $2 k R$.

structure at about $2 \mathrm{eV}$ from the first line is due to the next $1 s \rightarrow 5 p$ transition [7,9]. Above the ionization limit (indicated by the hatched lines), the absorption cross-section decreases monotonically. Note that the peak at $3225 \mathrm{eV}$ is due to multielectronic effects $[7,10]$. On the contrary, for the solid phase, the spectrum contains additional oscillations, which are due to the structure of the absorbing atom nearest neighbor. This oscillatory structure is characteristic of solids, liquids or molecular gases and its theory was originally proposed by Krönig in 1931 [11]. It was only worked out in detail after 1974 with the works of Stern et al. [12], Lee and Pendry [13] and Ashley and Doniach [14]. In the same time, it became a very fruitful and powerful experimental tool with the synchrotron radiation development. We will present here the main ideas for the understanding of these experiments (for more details, see for example Refs. 15,16).

For a photon energy well above the absorption edge, the ejected electron possesses a kinetic energy, $E$, which is approximately equal to $h v-E_{0}$. This energy is large compared with its interaction energy with the surrounding atoms and the photoelectron can be modeled by a free outgoing wave. This wave is scattered by the neighboring potentials and as a result, the final state is the superposition of the outgoing and scattered waves (Fig. 2). Single-scattering - i.e. scattering by only one atom is a first-order process and is generally the most important. The absorption cross-section is propor- tional to the square of the usual dipolar matrix element $M_{i f}=\left|\left\langle\psi_{i}|H| \psi_{f}\right\rangle\right|$, where $\psi_{i}$ is the initial state wave function, corresponding to the electron in the atomic core of the central atom and $\psi_{f}$ is the final state wave function, corresponding to the propagating photoelectron. The matrix element is non-zero only in the region where the core state is non-zero - that is, on the absorbing atom. It is thus only necessary to determine the final wave function at the center of the absorbing atom. The optical path length contributing to the phase shift between the outgoing and the backscattered waves is then $2 k R, R$ being the distance between the central atom and its scattering neighbor and $k$ the photoelectron wavevector. $k$ is given in a first rough approximation by the following formula: $\hbar^{2} k^{2} / 2 m=h v-E_{0}$, where $m$ is the mass of the electron. The waves will add or substract depending on the photoelectron energy and this will give rise to the EXAFS oscillations. The EXAFS spectrum is defined by $\chi=\left(\mu-\mu_{0}\right) / \mu_{0}$ and is obtained here after fitting the atomic absorption $\mu_{0}$ by a polynomial function of degree 3 and normalizing the data with the Lengeler-Eisenberger method [17]. The qualitative picture given above can be directly translated into an expression for $\chi$ and we will now comment the formula obtained:

$$
\begin{aligned}
& \chi=\sum_{i} \frac{F_{i}(k)}{k R_{i}^{2}} \sin \left(2 k R_{i}+\phi_{i}\right) \times \\
& \times \exp \left(-2 R_{i} / \lambda\right) \exp \left(-2 k^{2} \sigma_{i}^{2}\right),
\end{aligned}
$$

where $i$ designates the different neighbors of the central atom at distance $R_{i} ; F_{i}$ is the backscattering amplitude due to atom $i$, and $\phi_{i}$ contains the different phase shifts undergone by the electronic wave during first, its passage through the central atom $\left(\delta_{1}\right)$, second, to its back-scattering $\left(\varphi_{i}\right)$ and finally to its passage through the central atom, so that $\phi_{i}=2 \delta_{1}+\varphi_{i} . \lambda$ designates the electronic mean free path (a few $\mathrm{A}$ ), and the term $\exp \left(-2 R_{i} / \lambda\right)$ phenomenologically takes into account the decoherence of the outgoing and the scattered waves due to both the core and the photoelectron lifetimes. It represents the probability that the electron travels to the backscattering atom and returns without scattering or the core hole being filled. $\lambda$ contributes to the weakness of the far neighbor contribution in the EXAFS signal and is a function of $k$. Oscillations are also damped as a function of $k$ by the thermal disorder: noncorrelated movements of the central and the scattering atoms lead to a 
spreading of the instantaneous values of $R_{i}$, which can be modeled, for a low disorder and for a Gaussian distribution of the distances, by a factor of $\exp \left(-2 k^{2} \sigma_{i}^{2}\right)$, analogous to the Debye-Waller factor in diffraction experiments. $\sigma_{i}^{2}$ is the relative mean-square variation of the interatomic distance $R_{i}$ and can be approximated with the Debye model [18]. The introduction of a larger factor than that expected by thermal fluctuations may be necessary in order to fit the experimental damping and this reveals the existence of some additional static disorder.

The experimental spectrum $\chi$ is often weighted by $k$ in order to give more importance to its highfrequency part and Fig. $1, b$ shows the EXAFS spectrum $k \chi$ corresponding to the absorption of solid argon plotted in Fig. 1,a. In the following, we will plot $(k \chi)$ as a function of $E$ and, when necessary, spectra will be offset for clarity. Figure 1,c shows the Fourier transform corresponding to the EXAFS data of Fig. 1,b. A Kaiser window going from 2.1 to $8.7 \AA^{-1}$ (17 to $\left.290 \mathrm{eV}\right)$ has been used to calculate this Fourier transform. The different peaks emerging in Fig. 1,c at 3.3, 5.0, 6.2 and 7.2 $\AA$ correspond to the first, second, third and fourth shells of the argon atom's nearest neighbors and are very similar to previous results [8]. Remember that it is necessary to take into account the phase shift $\phi_{i}$ in order to get the actual distances in the crystal.

According to the EXAFS formula, the knowledge of electronic parameters such as $F_{i}, \phi_{i}$ and $\lambda$ allows to obtain structural parameters such as $R_{i}$, $\sigma_{i}^{2}$, and the number of neighbors. One way to obtain these electronic parameters is to calculate them by the FEFF code [19]. This code gives the contributions of both the single- and multiple-scattering processes by investigating all the different scattering paths. It then calculates their corresponding effective amplitude ( $F_{\text {eff }}$, from which FEFF takes its name) and effective phase shift $\left(\phi_{\text {eff }}\right)$ so that the total EXAFS spectrum can be readily obtained by adding all their contributions with the simple formula mentioned above. In the case of molecular species isolated in matrices, we are mainly interested in the intermolecular scattering, but we have to remember that this contribution will add the intramolecular one. We have always considered in the simulated EXAFS spectra all the singleand multiple-scattering paths whose length were less than $8 \AA$ and contributing more than $2.5 \%$. In the simulations concerning isolated molecules in argon or xenon, we used the Debye-Waller factor given by the FEFF program for a temperature of $15 \mathrm{~K}$ with a Debye temperature of $92 \mathrm{~K}$ for argon and $64 \mathrm{~K}$ for xenon. All the simulations presented in this paper have been done with the FEFF6 version of the code.

Figure $1, d$ shows the fit of the inverse Fourier transform of the first neighbor peak of Fig. 1,c with the amplitude and phase shift calculated by the FEFF program. A $\sigma^{2}$ factor of $0.024 \AA^{2}$ and a distance of 3.75(1) $\AA$ are obtained for this fit. This last value is in good agreement with previous determinations $[6,8]$ and gives an indication of the validity of the phaseshift as determined by the FEFF code. Let us recall here that the nearest neighbor's distance in argon (respectively xenon) is 3.76 (4.34) $\AA$ at $20 \mathrm{~K}$.

\section{Experimental}

When studying isolated molecules, the signal was recorded in a fluorescence mode, while it was in a transmission mode for pure argon. The first method allows a selective detection of the signal due to the absorbing molecule and is then more efficient for dilute samples. The gas mixture was deposited onto an $\mathrm{Al}$ foil stuck with silver print on the cold finger of a He cooled cryostat. The mixture was prepared outside the vacuum cell and introduced through a tube opening at about $2 \mathrm{~cm}$ in front of the cold sample holder with a rate of about $20 \mu \mathrm{mol} / \mathrm{min}$ so that the deposition conditions were similar to the usual ones. The concentration was $1: 100$ and the temperature was about $15 \mathrm{~K}$ during all the experiments. The thickness of the film was a few microns as can be estimated by the observation of visible interference fringes during the deposition. The basic pressure in the cell was $10^{-8}$ Torr and increased to $10^{-5}$ Torr during the deposition.

The photoabsorption measurements were carried out at the Laboratoire pour l'Utilisation du Rayonnement Electromagnetique (LURE-Orsay) on the SA32 beamline equipped with a double-crystal (InSb (111)) monochromator, which allows an energy resolution ranging from about $0.7 \mathrm{eV}$ for a photon energy of $1800 \mathrm{eV}$ to about $1.4 \mathrm{eV}$ at $3500 \mathrm{eV}$. The SuperACO storage ring was operating at $800 \mathrm{MeV}$, with a typical current of $200 \mathrm{~mA}$. The incident beam was monitored by measuring the total electron drain current of a polyurethane foil covered by $80 \mathrm{~nm}$ of $\mathrm{Ti}$, located downstream the monochromator. The focused spot on the sample was about $500 \mu \mathrm{m}$ horizontally and $300 \mu \mathrm{m}$ vertically. The energy calibration was performed by taking the $K$ edge of a sample of $\mathrm{ZnS}$ at $2472 \mathrm{eV}$. The absorption spectra at the $K$ edge of silicon $(1839 \mathrm{eV})$, sulfur $(2472 \mathrm{eV})$, chlorine $(2833 \mathrm{eV})$ 
and argon $(3206 \mathrm{eV})$ were collected in the fluorescence mode as a function of the incident photon energy with a $1 \mathrm{eV}$ step. We recorded several scans for each sample so that the total collecting time for one point was ranging from 10 to $20 \mathrm{~s}$. The fluorescence yield was measured at $90^{\circ}$ from the incident $\mathrm{x}$-ray beam by a seven-element Ge detector (Eurisys-Mesures) for the $K_{\alpha_{1}} / K_{\alpha_{2}}$ lines, with an energy resolution better than $150 \mathrm{eV}$. In the case of pure solid argon, the set-up was different and the direct absorption spectrum through argon deposited onto a thin $\mathrm{Al}$ foil has been recorded with a silicon diode detector.

\section{Results and discussion}

\subsection{Probing the host material: OCS isolated in argon, xenon, nitrogen and methane matrices}

Figure 3 shows the absorption coefficient $\mu$ displayed as a function of the photon energy from 2450 to $2600 \mathrm{eV}$ at the $K$ edge of the sulfur atom in the case of argon, xenon, nitrogen and methane matrices. The pre-edge contribution has been subtracted for each spectrum after its fit by a linear regression, except for xenon. For this latter case, the signal was much weaker than for the other cases because the

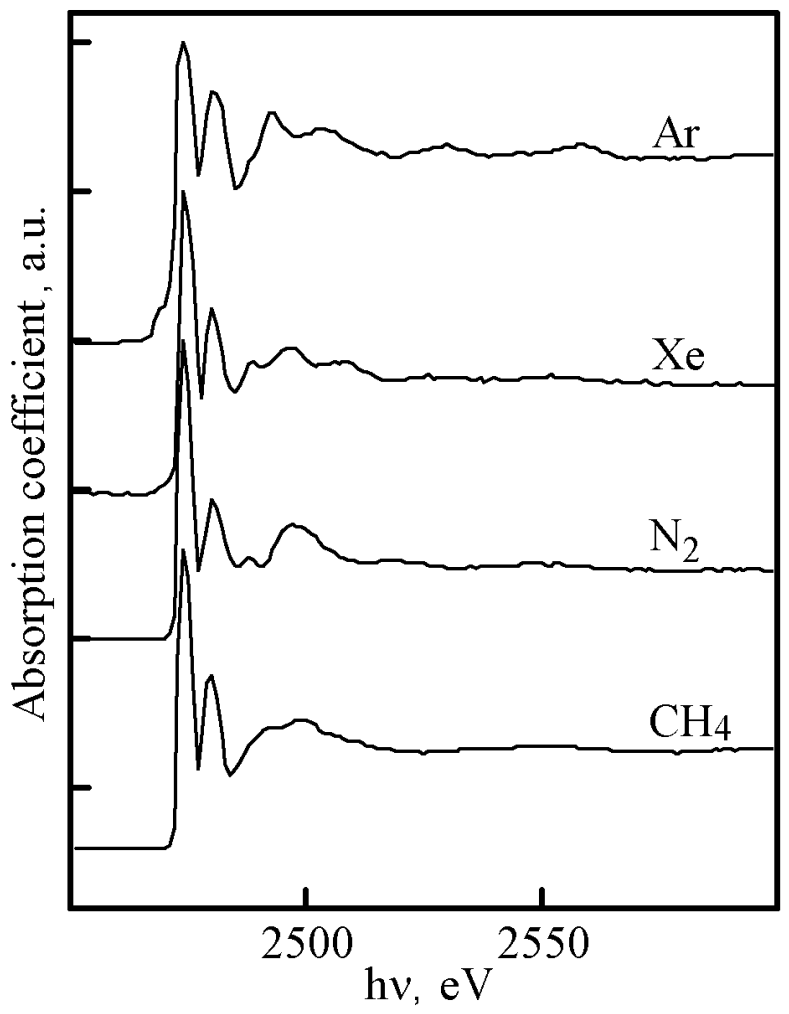

Fig. 3. X-ray absorption spectra at the $K$ edge of $\mathrm{S}$ for OCS isolated in argon, xenon, nitrogen and methane matrices. The fluorescence signals have been normalized to unity at the maximum of the first line.

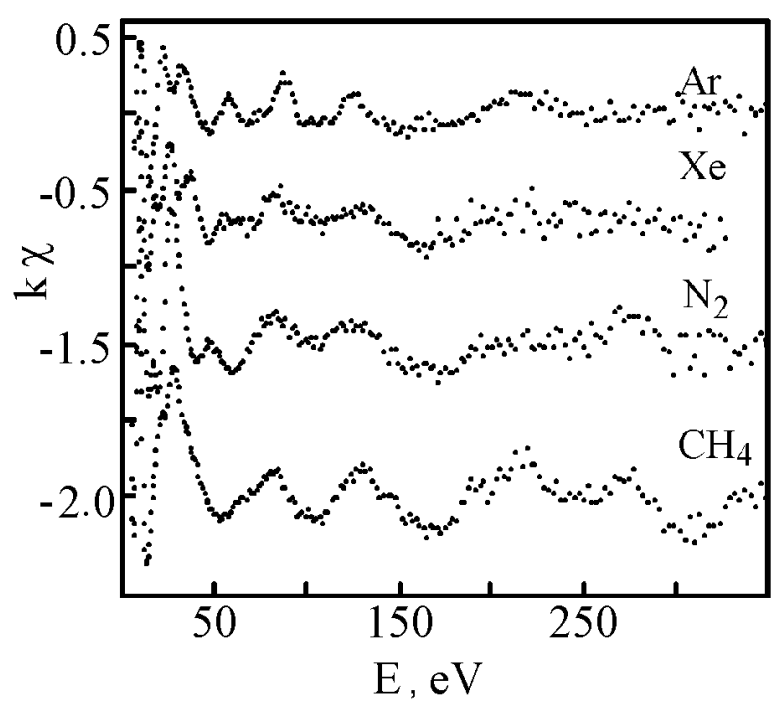

Fig. 4. EXAFS oscillations $k \chi$ of the spectra of Fig. 3 as a function of the photoelectron energy. The spectra have been shifted for a sake of clarity but the scale is exact for their amplitudes.

absorption of the fluorescent photons by the matrix itself reduces their escape depth. Such a low-level signal frequently induces a perturbed profile for the data: in order to correct it on this figure, we have subtracted a fit by a polynomial function of degree two of the whole data above the edge.

The corresponding EXAFS spectra $(k \chi=$ $\left.=k\left(\mu-\mu_{0} / \mu_{0}\right)\right)$ are displayed in Fig. 4 as a function of the photoelectron's kinetic energy. Oscillations are clearly visible up to $300 \mathrm{eV}$ above the absorption edge.

A close look at Fig. 4 shows some similarity on the high-energy part of the oscillations (from 80 to $350 \mathrm{eV}$ ) especially for the $\mathrm{Xe}, \mathrm{N}_{2}$ and $\mathrm{CH}_{4}$ matrices. The following questions are then arising: do these spectra actually contain information about the OCS environment? Don't they only reveal the own structure of OCS? Moreover, the concentration of $1: 100$ is indeed high enough for clustering to occur and spectra may also only reflect the structure of some aggregates. As a matter of fact, infrared measurements performed on OCS isolated in nitrogen, in argon or in xenon [20,21] reveal that clustering and/or multiple trapping sites were prevalent under all but the most dilute conditions $(1 / 50000)$ and so we will examine now in detail if there is evidence for a signature of the isolation in our spectra.

The same data are given with an extended scale $(10-100 \mathrm{eV})$ in Fig. 5 in order to focus on the low-energy part of the spectra. Significant differences are observed on the oscillations, reflecting 


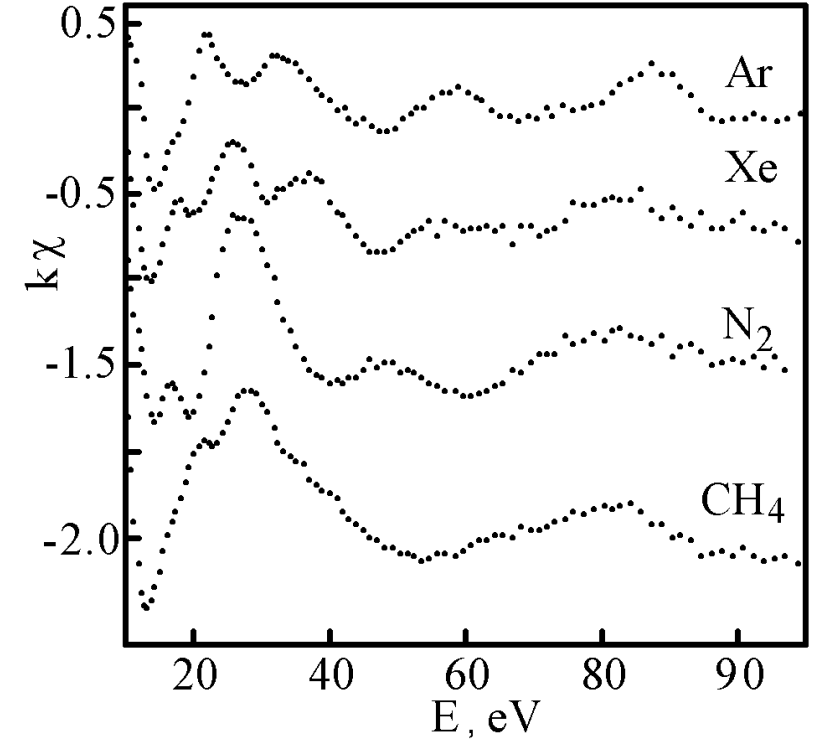

Fig. 5. The same as Fig. 4 with an extended scale.

differences in the absorbing sulfur atom environment. This proves that, whatever the exact rate of clustering in the matrix is, a signal corresponding to the isolated molecule and thus, information about the molecular cage structure, is unambiguously present in these measurements.

In order to clear up this point, we have recorded the EXAFS spectrum of pure OCS deposited under the same conditions. It is represented in Fig. 6 and is compared with the data concerning OCS isolated in methane. We recognize once more the same high-energy oscillations and the question of clustering rises again. We have also done an FEFF simulation of solid OCS based on the $R 3 m\left(C_{3 v}^{5}\right)$ crystal

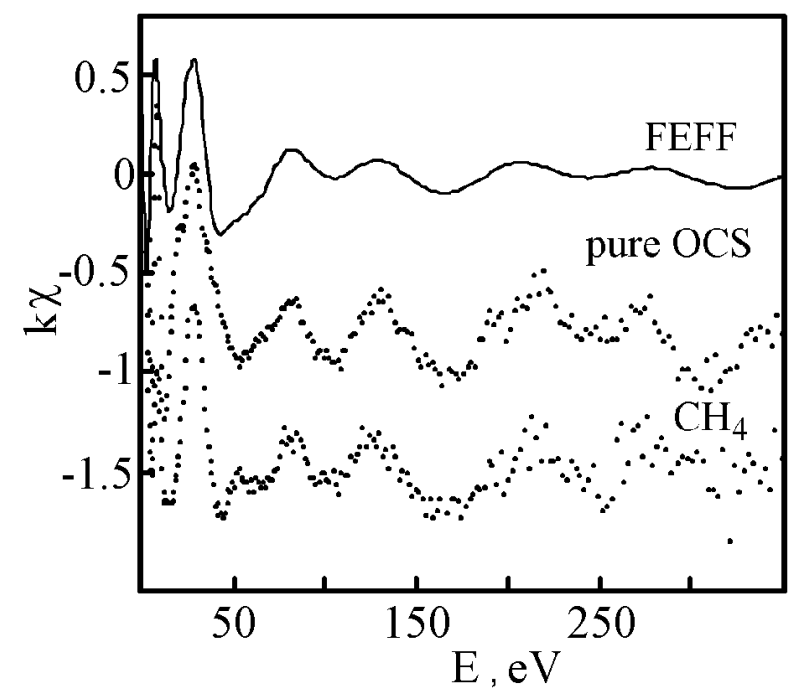

Fig. 6. The same as Fig. 4 for OCS isolated in methane, pure OCS and FEFF simulations of the OCS molecular single scattering signal.

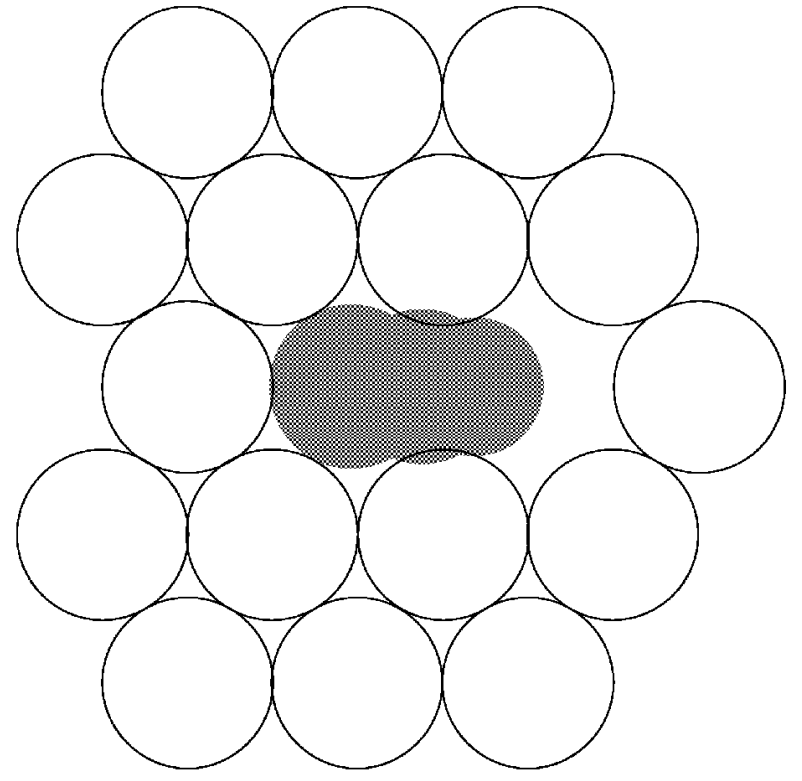

Fig. 7. Scheme of the two-hole trapping site for OCS in an argon matrix.

structure determined by neutron diffraction [22]. We have extracted from the calculation the EXAFS contribution of the excited OCS molecule by selecting only the intramolecular single diffusion paths and this result is added in Fig. 6. It is clear that the resulting periods of the oscillations fit those of the experimental spectra and we can conclude that the high-energy structures appearing for OCS isolated in $\mathrm{Xe}, \mathrm{N}_{2}$ and $\mathrm{CH}_{4}$ are mainly due to intramolecular $\mathrm{S}-\mathrm{C}$ and $\mathrm{S}-\mathrm{O}$ scattering.

Let us explain now why the matrix signature is mainly present only in the first part of the spectra. A general reason is the damping induced by the thermal excitation of atomic movements: as a matter of fact, intramolecular bonds are stiffer than intermolecular bonds and the $\sigma^{2}$ factor is much lower for a pair of atoms both belonging to the molecule than for a sulfur-argon pair. In the case of $\mathrm{CH}_{4}$ and $\mathrm{N}_{2}$, a second reason is the well-known poor scattering efficiency of the low- $Z$ atoms. In addition, the backscattering amplitudes decrease as the photoelectron kinetic energy increases, the more rapidly the lighter the element is. For example, the maximum of the backscattering amplitude is found for kinetic energy of about $20 \mathrm{eV}$ in the case of $\mathrm{N}$, while it is about $90 \mathrm{eV}$ in the case of Ar. These reasons explain that we do not measure a significant contribution coming from the $\mathrm{N}$ or $\mathrm{C}$ neighbors for an energy larger than $50 \mathrm{eV}$.

The IR spectrum and the trapping site for OCS isolated in argon and xenon have been modeled by Winn [23]. The molecule is found to accommodate a two-hole site as represented schematically in 


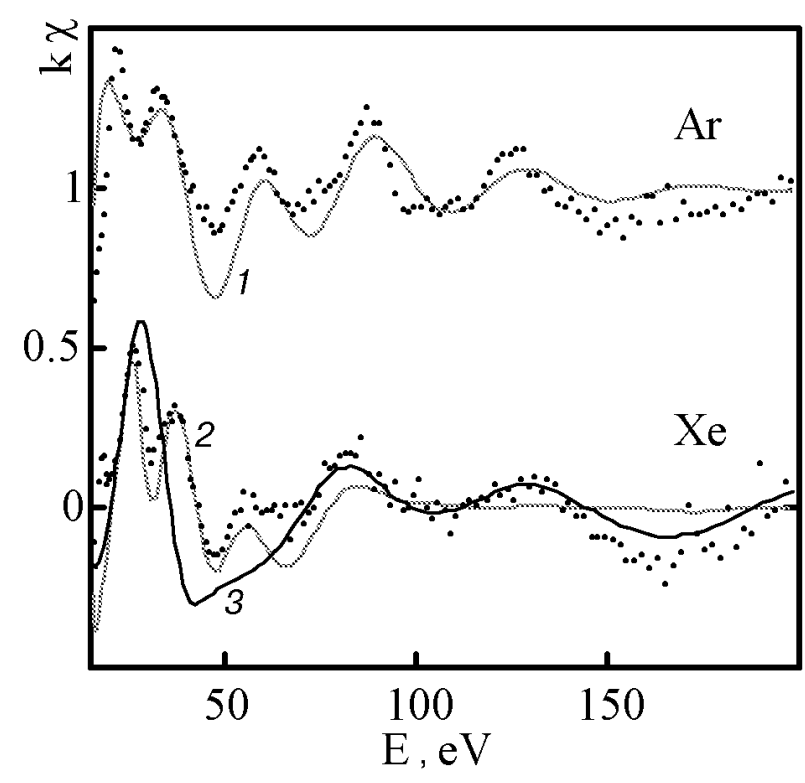

Fig. 8. EXAFS oscillations (dots) and FEFF simulations of the two-hole site of Ref. 23 for OCS isolated in argon (1) and in xenon (2). In the latter case, the additional line 3 is the FEFF simulation of molecular OCS as in Fig. 6.

Fig. 7. In the case of argon, the sulfur atom is roughly at the center of one of the two holes and the neighboring argon atoms occupy different welldefined positions with $\mathrm{S}-\mathrm{Ar}$ distances spreading around a mean value of $3.74 \AA$. In the case of xenon, in addition to this site, a second where the oxygen atom is at the center of one of the two holes has been found to be unstable. Figure 8 compares the EXAFS signals of OCS isolated in argon or in xenon with FEFF simulations performed with the geometry deduced from Ref. 23 and corresponding to Fig. 7 - the sulfur atom being about at the center of a substitutional hole. Concerning the xenon matrix, the FEFF results corresponding to the unstable site are not represented here for the sake of clarity, but they clearly do not agree with the experimental signal. On the contrary, Fig. 8 shows that the oscillations corresponding to the calculated stable site agree well with the three first main structures observed between 20 and $100 \mathrm{eV}$. The line 3 corresponds to the EXAFS simulations of the OCS molecule alone as for Fig. 6, and it fits well the oscillations between 100 and $250 \mathrm{eV}$. This shows that the $\mathrm{S}-\mathrm{Xe}$ contribution is only visible up to $100 \mathrm{eV}$ - as a matter of fact, the backscattering amplitude rapidly decreases above $90 \mathrm{eV}$ - and confirms that the following part of the spectrum is dominated by the pure OCS contribution. In the case of argon, it seems clear that the main oscillating period obtained with the calculations is larger than the experimental one and this reveals that the measured mean $\mathrm{S}-\mathrm{Ar}$ distance is larger than the
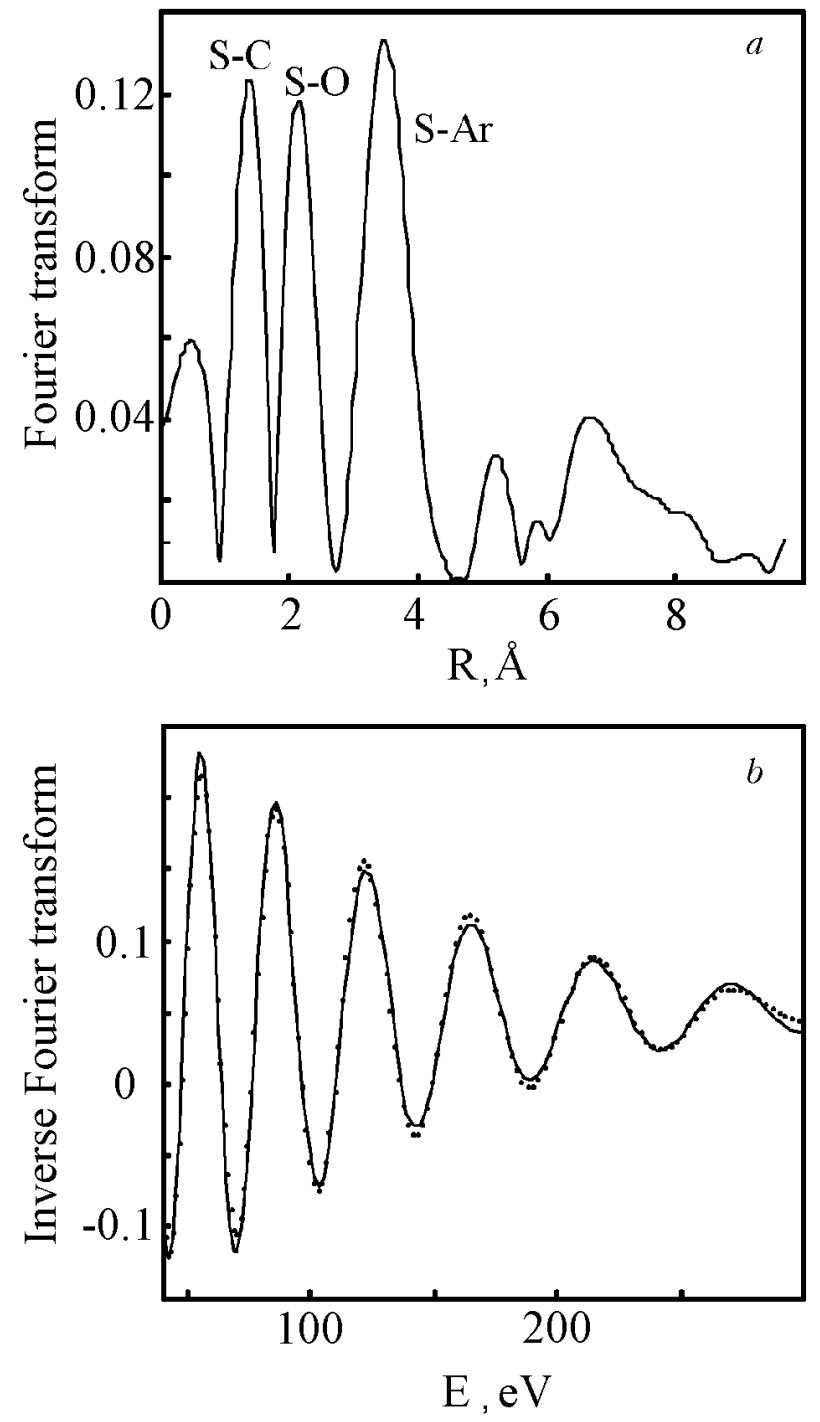

Fig. 9. OCS isolated in argon [24]. (a) Fourier transform of the EXAFS oscillations represented in Fig. 4; $(b)$ inverse Fourier transform: the dots correspond to the experimental data and the continuous line corresponds to a fit with the electronic parameters given by the FEFF code.

calculated one. On the other hand, we can observe that the sulfur-rare gas contribution is more dominant for argon than for xenon and then, the OCS / Ar system appears the best candidate for a quantitative analysis of the signal.

This has been done in a previous paper [24] and the results are summarized now. The Fourier transform of the EXAFS spectrum obtained for OCS in argon (Fig. 9,a) shows three peaks corresponding to $\mathrm{S}-\mathrm{C}, \mathrm{S}-\mathrm{O}$ and $\mathrm{S}-\mathrm{Ar}$ distances so that the structure of the first shell of argon atoms surrounding OCS can be analyzed by fitting the oscillations given by the inverse Fourier transform of the peak corresponding to the $\mathrm{S}-\mathrm{Ar}$ distance (Fig. 9, $b$ ). We found that our results were consistent with a shell of 11 argon atoms at a mean distance of 3.78(1) $\AA$, larger than the pure Ar-Ar distance found with the same 
FEFF data (3.75(1) ̊). A static disorder around this position has been measured by an additional Debye-Waller factor of $\sigma^{2}=0.006 \AA^{2}$, which corresponds to a root mean square displacement of about $0.08 \AA$. This disorder is moderate and this is in good agreement with the measured relaxation of the argon positions in order to accommodate the molecule. These parameters for the cage are also in agreement with the two-hole site picture represented in Fig. 7 and they only slightly differ from the perfect cage structure calculated by Winn [23]: they correspond to a structure which is less organized (a unique mean S-Ar distance instead of a set of well-defined positions) and to a larger mean S-Ar distance (3.78 $\AA$ instead of $3.74 \AA$ ). We must remark that the concentration of our sample (1/100) is high enough so that our matrix crystal is probably disturbed by the OCS dopant and it is then not surprising to obtain a structure that differs from the perfect one.

\subsection{Probing the distortion induced by the guest molecule: $\mathrm{OCS}, \mathrm{HCl}$ and $\mathrm{SiH}_{4}$ isolated in an argon matrix}

The absorption spectra of $\mathrm{SiH}_{4}$ and $\mathrm{HCl}$ isolated in an argon matrix at the $K$ edge of the silicon and the chlorine atom, respectively, have been recorded and are reported in Fig. 10,a. For comparison, the spectrum of OCS in argon at the sulfur $K$ edge, already presented in the preceding section, is reproduced in the same figure, as well as the spectrum of pure argon recorded at the argon $K$ edge. This last spectrum can be thought here as Ar in Ar. A shift of the photon energy corresponding to the $K$ electron binding energy $E_{0}$ is applied for each spectrum in order to be able to compare them, so that the abscissa is $h v-E_{0}$ and roughly corresponds to the photoelectron kinetic energy. The oscillations are observed above the absorption edge in the cases of OCS, $\mathrm{HCl}$ and $\mathrm{Ar}$, while they are much more rapidly damped in the case of $\mathrm{SiH}_{4}$. Moreover, when existing, they undoubtedly show a similarity, especially in the 40-150 eV region (Fig. 10,b), which proves that they are the signature of a similar argon environment. The peak existing for $\mathrm{HCl}$ only, at about the origin of the energy scale of Fig. 10, $a$, corresponds to the transition towards the molecular antibonding $\sigma^{*}$ state [25]. The following peak can be readily compared for $\mathrm{HCl}$ in argon and for pure argon: it corresponds to the $1 s \rightarrow 4 p$ Rydberg transition $[8,25]$, which is expected to be very similar in both cases because of similar electronic structures.

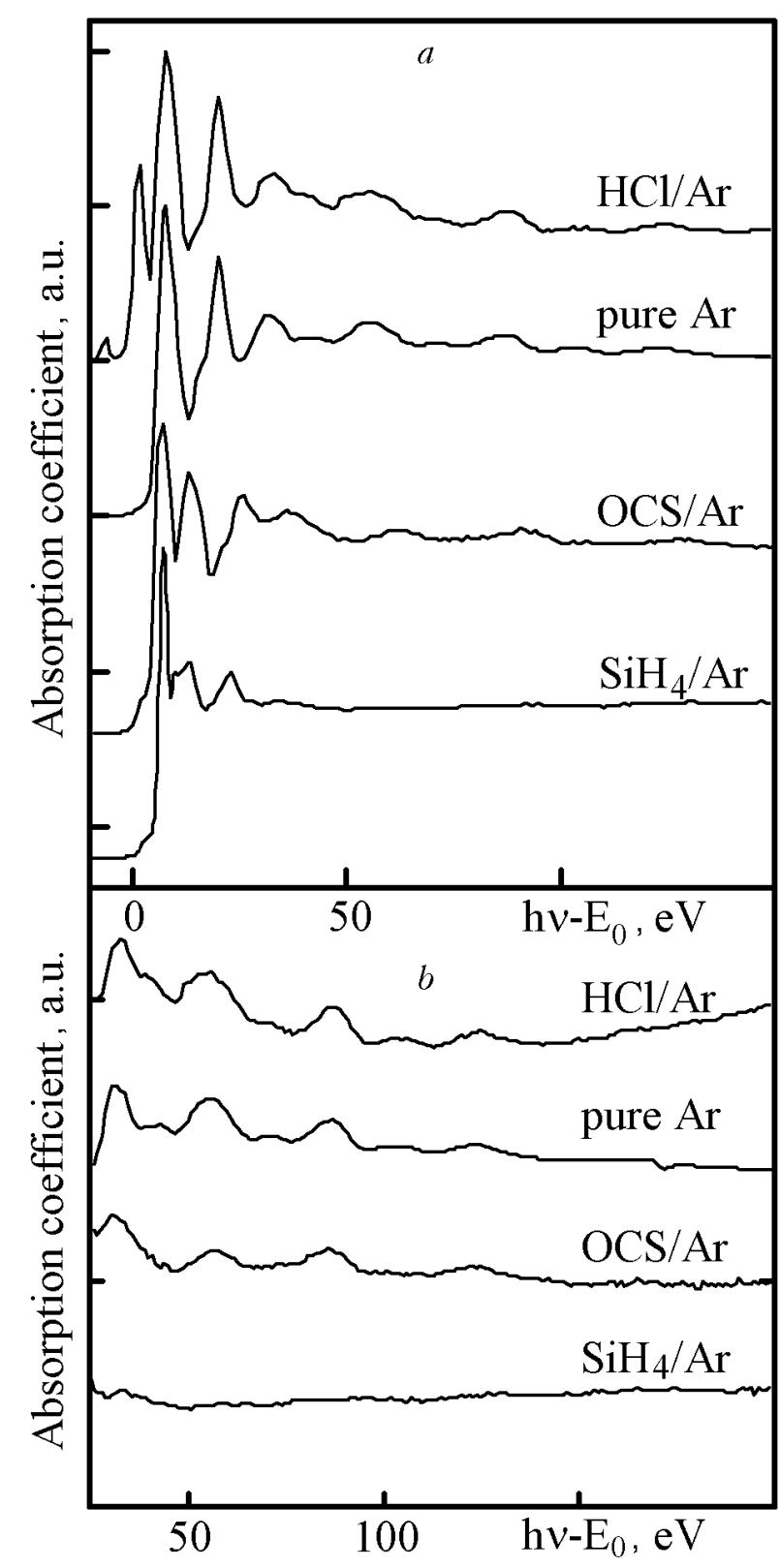

Fig. 10. X-ray absorption spectra as a function of the shifted photon energy ( $\left.h v-E_{0}\right), E_{0}$ being the $K$ edge energy of $\mathrm{Cl}$ for $\mathrm{HCl}, \mathrm{S}$ for OCS and Si for $\mathrm{SiH}_{4}$ (fluorescence mode) and the $K$ edge of Ar for pure argon (transmission mode). In the case of $\mathrm{HCl}$, the spectrum has been corrected to eliminate an important unwanted structure at $45 \mathrm{eV}$ due to a glitch: (a) spectra from -10 to $150 \mathrm{eV} ;(b)$ the same spectra in an extended range.

The EXAFS signals $(k \chi)$ for pure argon and for $\mathrm{HCl}$ isolated in argon are superimposed in Fig. 11 and no significant difference is observed between the two spectra. This confirms that a single substitutional site readily accepts $\mathrm{HCl}$ without perturbation of the crystalline structure. The size of the molecule is actually close to the size of the argon atom and moreover, it is well-known that the molecule undergoes almost free rotation in the cage, 


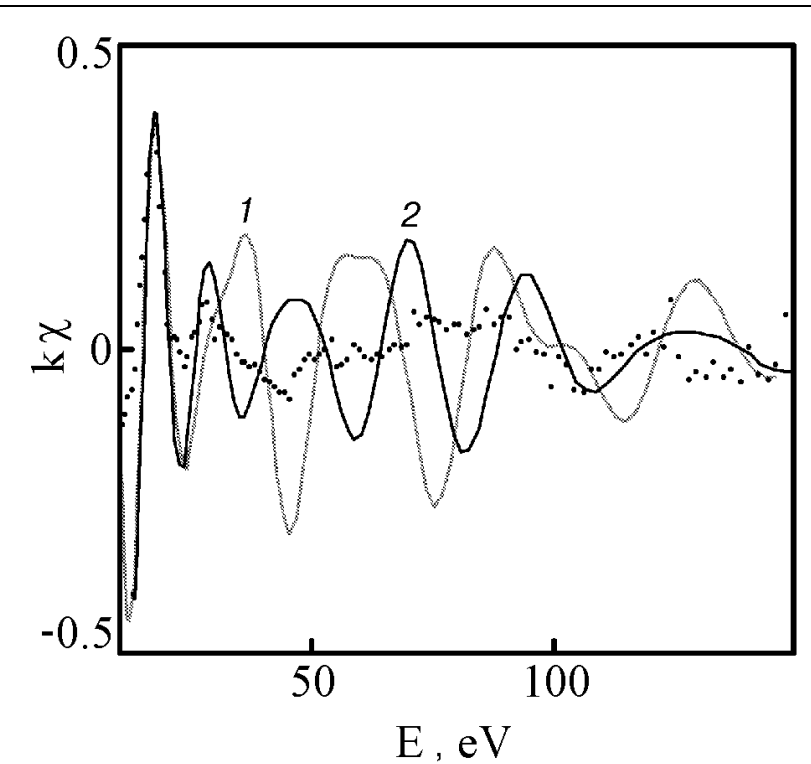

Fig. 11. EXAFS spectra $(k \chi)$ as a function of the photoelectron energy of pure argon (continuous line) and of $\mathrm{HCl}$ (dots) isolated in argon.

indicating a negligible hindering $[26,27]$ by the neighboring atoms. The beating between different oscillations that is observed in the spectra (see Fig. 11) is due to the addition of the contributions of the different shells of argon surrounding the excited atom and of multiple scattering. The detailed analysis of the different path contributions given by the FEFF code shows that the main oscillating structure corresponds to the sine function built with the first nearest neighbors at a distance of $3.75 \AA$ while the secondary maxima appearing at $E=67$ and $100 \mathrm{eV}$ are due to a constructive addition of the third and fourth shell sine functions and of the double scattering contributions, especially those coming from two Ar scattering atoms aligned with the central atom. This latter signal involves indeed a forward scattering and is enhanced because of its high amplitude (focusing effect).

On the contrary, silane, whose tetrahedral symmetry is well-suited for a cubic crystal, does not give rise to long-range oscillations. Infrared spectroscopy [28,29] reveals two peaks for the $\mathrm{Si}-\mathrm{H}$ stretching band of silane isolated in argon, indicating two sites for its accomodation. The mean lowfrequency peak arises over a very broad and structured band due either to rotation or to multiple configurations of the site. Silane is approximately a sphere whose diameter is $5 \AA$ and is probably too large to fit into a one-atom vacancy of an argon crystal. It will neither easily occupy a two-atom one (Fig. 7), which can accommodate only a prolate molecule of reduced size. We have also considered

Fig. 12. EXAFS spectrum $(k \chi)$ of silane isolated in argon (dots) and FEFF simulations of silane in a one-hole site (line 1 ) and in a four-hole site (line 2).

the case of the four-atom vacancy of tetrahedral symmetry as it was done for some metallic atoms [30]. In Fig. 12, we show the superimposition of the FEFF results obtained for silane in a one-atom vacancy, for silane in a four-atom vacancy, and of the experimental data. There is clearly no agreement between the oscillating structures of the simulations and the experimental results and we cannot conclude on the validity of any of the two sites. Nevertheless, the experimental spectrum may also result from the addition of two signals as suggested by the infrared results. Unfortunately, EXAFS oscillations are expanding in a too short range to give any valuable information by the usual Fourier transform operation. We can only conclude that the mean environment of silane do not present enough simplicity to give rise to long range EXAFS oscillations.

\section{Conclusion}

We have presented here the EXAFS experiments performed at the $K$ edge of $\mathrm{Si}, \mathrm{S}$ and $\mathrm{Cl}$ for $\mathrm{SiH}_{4}$, OCS and $\mathrm{HCl}$ isolated in argon and for OCS isolated in argon, xenon, nitrogen and methane. The signature of the different matrix environments of OCS is clearly evidenced in the low-frequency part of the spectra and, on the other hand, the signature of molecular OCS is dominant in the high-frequency part, except in the case of the argon matrix. As a matter of fact, to obtain a signal containing non-negligible intermolecular contribution, the host atom must not only have a good 
scattering efficiency in order to get a large amplitude for the oscillations (and so, not a low- $Z$ atom) but must also weakly absorb the $\mathrm{x}$-ray radiation in order to get a good signal-to-noise ratio in the fluorescence detection mode (and so, not a high- $Z$ atom).

For this reason, we have been able to do a complete analysis of the EXAFS oscillations only in the case of OCS in argon. We have measured an S-Ar of $3.78 \AA$ and we have shown that this slight relaxation of the argon cage was enough to accommodate the molecule without perturbing the longrange order of the matrix. These results are consistent with the usual two-hole site picture invoked for such a linear molecule and are not far from a previous modeling [23] valid anyway only for a perfect matrix. For OCS in xenon, the results are entirely consistent with the same modeling and confirm the calculated location of OCS inside the two vacancies. The case of silane in argon is totally different and only a highly damped signal has been measured so that, in agreement with previous infrared results, we can exclude the hypothesis of a single well-defined cage around the molecule and even the hypothesis of a disordered one-hole or four-hole site.

In conclusion, we think that probing the matrix environment of a diluted sample with the EXAFS technique in this soft $\mathrm{x}$-ray region is not a simple task because of the poor signal-to-noise ratio and of the problem of the absorption by the matrix itself. Nevertheless, consistent results have been obtained here and this proves that the technique can really give important information about the site structure and, even if a quantitative analysis is not always possible, that it can provide valuable information about the ordering around the molecule.

1. C. S. Barret and L. Meyer, J. Chem. Phys. 41, 1078 (1964).

2. Y. Sonnenblick and Z. H. Kalman, J. Cryst. Growth 58, 143 (1982)

3. W. Langel, W. Schuller, E. Knözinger, and H.-W. Fleger, J. Chem. Phys. 89, 1741 (1988); A. Becker, W. Langel, S. Maass, and E. Knözinger, J. Phys. Chem. 97, 5525 (1993).

4. N. A. Young and M. D. Spicer, J. Mol. Struct. 222, 77 (1990); I. R. Beattie, P. J. Jones, and N. A. Young, J. Am. Chem. Soc. 114, 6146 (1992); N. A. Young, J. Chem. Soc. Dalton Trans. 249 (1996).

5. P. A. Montano and G. K. Shenoy, Solid State Commun. 35, 53 (1980); P. A. Montano, W. Schulze, B. Tesche, G. K. Shenoy, and T. I. Morrison, Phys. Rev. B30, 672 (1984).
6. W. Malzfeldt, W. Niemann, P. Rabe, and R. N. Schwentner, in Exafs and near Edge Structure, A. Bianconi, I. Incoccia, and S. Stipcich (eds.), Springer, Berlin (1983); W. Malzfeldt, W. Niemann, P. Rabe, and R. Haensel, in Exafs and near Edge Structure III, K. O. Hogson, B. Hedman, and J. E. Penner-Hahn (eds.), Springer, Berlin (1984).

7. R. D. Deslattes, R. E. LaVilla, P. L. Cowan, and A. Henins, Phys. Rev. A27, 923 (1983); R. D. Deslattes, Aust. J. Phys. 39, 845 (1986).

8. E. Rühl, C. Heinzel, A. P. Hitchcock, H. Schmelz, C. Reynaud, H. Baumgärtel, W. Drube, and R. Frahm, J. Chem. Phys. 98, 6820 (1993).

9. M. Breinig, M. H. Chen, G. E. Ice, F. Parente, and B. Crasemann, Phys. Rev. A22, 520 (1980).

10. M. Deutsch, N. Maskil, and W. Drube, Phys. Rev. A46, 3963 (1992)

11. De L. Kronig, Z. Phys. 70, 317 (1931).

12. D. E. Sayers, F. W. Lytle, and E. A. Stern, Phys. Rev. Lett. 27, 204 (1971); E. A. Stern and D. E. Sayers, Phys. Rev. Lett. 30, 174 (1973); E. A. Stern, Phys. Rev. B10, 3027 (1974); E. A. Stern, Phys. Rev. B10, 3027 (1974); E. A. Stern, D. E. Sayers, and F. W. Lytle, Phys. Rev. B11, 4836 (1975).

13. P. A. Lee and J. B. Pendry, Phys. Rev. B11, 2795 (1975).

14. C. A. Ashley and S. Doniach, Phys. Rev. B11, 1279 (1975).

15. P. A. Lee, P. H. Citrin, P. Eisenberger, and B. M. Kincaid, Rev. Mod. Phys. 53, 769 (1981).

16. X-ray Absorption, Principles, Applications, Techniques of EXAFS, SEXAFS and XANES, D. C. Koningsberger and R. Prins (eds.), John Wiley and sons, New-York (1987).

17. B. Lengeler and P. Eisenberger, Phys. Rev. B21, 4507 (1980).

18. G. Beni and P. M. Platzmann, Phys. Rev. B14, 1514 (1976).

19. J. J. Rehr, J. Mustre de Leon, S. I. Zabinsky, and R. C. Albers, J. Am. Chem. Soc. 113, 5135 (1991); J. Mustre de Leon, J. J. Rehr, S. I. Zabinsky, and R. C. Albers, Phys. Rev. B44, 4146 (1991).

20. F. D. Verderame and E. R. Nixon, J. Chem. Phys. 44, 43 (1966).

21. V. I. Lang and J. S. Winn, J. Chem. Phys. 94, 5270 (1991).

22. J. S. W. Overell, G. S. Pawley, and B. M. Powell, Acta Cryst. B38, 1121 (1982).

23. J. S. Winn, J. Chem. Phys. 94, 5275 (1991).

24. P. Roubin, S. Varin, C. Crepin, B. Gauthier-Roy, A.-M. Flank, R. Delaunay, M. Pompa, and B. Tremblay, $J$. Chem. Phys. 109, 7945 (1998).

25. S. Bodeur, J.-L. Marechal, C. Reynaud, D. Bazin, and I. Nenner, Z. Phys. D17, 291 (1990).

26. D. Maillard, A. Schriver, and J.-P. Perchard, J. Chem. Phys. 71, 505 (1979).

27. M. Allavena, H. Chakrouna, and D. White, J. Chem. Phys. 77, 1757 (1982).

28. R. E. Wilde, T. K. K. Srinivasan, R. W. Harral, and S. G. Sankar, J. Chem. Phys. 55, 5681 (1971).

29. N. Legay-Sommaire and F. Legay, J. Phys. Chem. 102, 8759 (1998)

30. M. E. Fajardo, J. Chem. Phys. 98, 119 (1993); J. A. Boatz and M. E. Fajardo, J. Chem. Phys. 101, 3472 (1994). 